\title{
Design Methods for Miniature Underwater Soft Robots
}

\author{
Frank Bonnet, Norbert Crot, Daniel Burnier and Francesco Mondada
}

\begin{abstract}
A robotic fish accepted by animals as conspecifics is a very powerful tool in behavioral biology. To obtain the acceptance of the device towards the animals, it should generate some visual stimuli that can be perceived by a living fish as attractive signals. In this paper we introduce a novel design of a robotic fish lure based on a Rigid-Flex Printed Circuit Board (PCB) for experiments on the fish collective behavior. The 3D scan of a zebrafish Danio Rerio, a model animal for fish behavior studies, was used to mimick the main visual features of the zebrafish on the design. The robot can generate different stimuli thanks to its actuated caudal peduncle and two LightEmitting Diodes (LEDs) placed near its eyes. The prototype has a total length of $63 \mathrm{~mm}$ and is only 1.5 times bigger than a zebrafish. The final prototype is waterproof and functional and thus satisfies the necessary conditions for the next steps that will be interaction experiments between the device and living zebrafish.
\end{abstract}

\section{INTRODUCTION}

Many example of social fish studies involving robotized fish lures can already be found in the scientific literature. Some of these devices are totally passive underwater as for example in [1]-[5], where dummy fish that mimick some of the fish body features are driven using a mobile robot that is moving outside of an experimental tank. Some other studies are using robotic artifacts in water [6]-[8] that allows them to generate more types of stimuli such as a beating tail or electric signals, and thus vary the attractiveness of the device towards the fish.

The design of such a robot dedicated to experiments with fishes have to originate from relevant sensory modalities and behaviors of the animal under study [8]. Therefore, a good understanding of the animal biology and its behaviors must precede the robot design process. The zebrafish Danio Rerio is a social fish and one of the most important vertebrate model organisms in genetics, developmental biology and biomedicine, therefore it is often selected as a model to conduct experiments on fish social interactions. The shoaling behavior is believed to be innate and it starts soon after hatching. Shoaling decisions in fishes often depend on the phenotypic characteristics of group members (such as body size or color) [10] and, in the case of the zebrafish, experimental results showed that individuals show preference towards their own conspecifics, with the color, body shape and stripes playing an important role [11]-[12]. Finally, some studies that involved interaction between robotic lures and zebrafish showed a variation in the acceptance of the robotic

\footnotetext{
${ }^{1}$ Frank Bonnet, Norbert Crot, Daniel Burnier and Francesco Mondada are with the Robotic Systems Laboratory, School of Engineering, Ecole Polytechnique Fédérale de Lausanne, Lausanne, Switzerland frank.bonnet@epfl.ch
}

device among a shoal of living zebrafish depending on the beating frequencies, color, noise and aspect ratio [8].

We designed in previous years a robotic fish lure that is equipped with an actuated caudal peduncle [13] (Fig 1). This lure can follow a group of living animals using an external wheeled mobile robot that is coupled with the robotic lure using magnets. We showed that the lure could attract a shoal of five zebrafish inside of a circular corridor with a certain effect, that depended mainly on the speed of the device, but this effect was still not enough to guarantee a full integration of the lure within the fish school. We believed that it was mainly due to the big size of the lure as its length was $80 \mathrm{~mm}$ thus with a ratio more than 1.8 as zebrafish, and to its shape that was not exactly mimicking the features of a zebrafish. The device was also not convenient to use for biologists, as it was not reprogrammable.

Consequently, we created a smaller design based on the 3D scan of a zebrafish and including a new stimulus, a RedGreen-Blue (RGB) Light emitted Diode (LED). The new design will also allow the user to reprogram the device once it is molded inside of the waterproof polyurethane coating which was not possible with the previous design. Another advantage of a smaller design is the reduction of the mass, which will reduce the inertia of the system and therefore allow more dynamic motions underwater. In order to achieve a smaller design while adding new electronic components and conserving the zebrafish body features, we used a Rigid-Flex Printed Circuit Board that, once folded, offer more available surfaces in order to fit the electronic components and routing, while decreasing the overall volume of the system. We also had to develop some soldering and folding techniques due to the small size of the selected electronic components that did not allow to use conventional techniques.

The paper is organized as follows: Section II provides the specification that we used to design the new version of the robotic lure; in Section III we describe the hardware design of the robot and in Section IV the assembly step that includes the folding of the Rigid-Flex PCB and the molding of the different parts to guarantee the waterproofness of the device. Section V presents the validation of the system and, finally, Section VI concludes the paper.

\section{SPECIFICATIONS}

The design of a miniature and underwater robot is always of great challenge. The device should be waterproof, wireless and, moreover in the case of this study, the size of the robot should be in the range of a zebrafish that rarely exceed 40 $\mathrm{mm}$ of length [14]. 


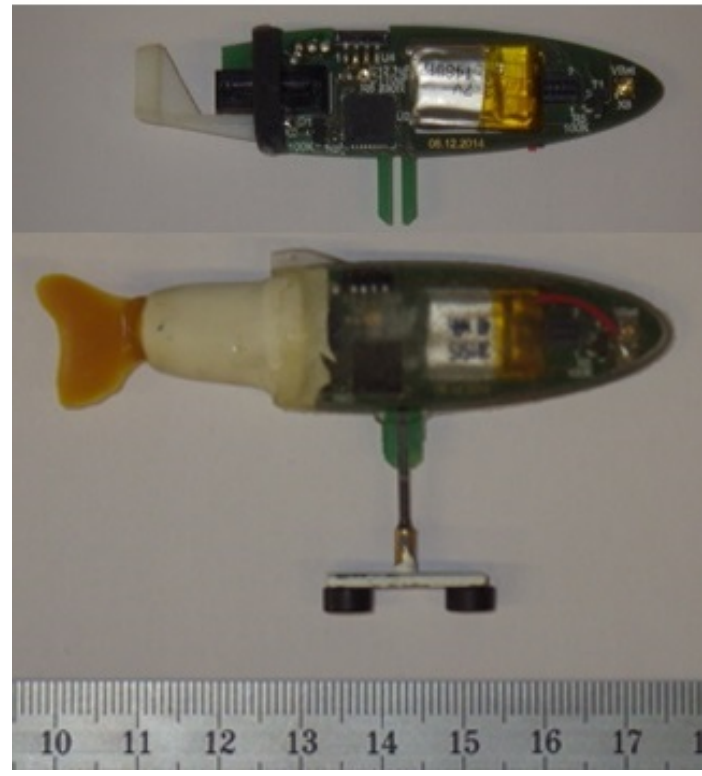

Fig. 1. The preliminary robotic lure design. Top: PCB with components mounted. Bottom: PCB molded into Polyurethane and Latex. The small module underneath the lure is composed of an iron plate, a carbon stick and two small magnets. This module is used to couple the lure with an external mobile robot moving underneath the tank (Units in [mm]).

Based on the lessons learned from the first design, the preliminary experiments obtained with zebrafish and the known zebrafish stimuli perception, we draw up the following specifications for the robot: The dimensions of the lure need to be reduced and as close as possible to a real zebrafish. It should have a better biomimetic appearance, by adding more realistic fins and body features. The robot has to integrate RGB LEDs in order to generate a new type of stimulus never tested on zebrafish with such robotic lures, as only different color surfaces were tested in published studies. Finally, the device should be reprogrammable once molded which was not the case for the first design.

\section{HARDWARE DESIGN}

\section{A. Rigid-Flex PCB design}

In the previous design, a two-copper-layer PCB was used as a skeleton (Fig. 1). This PCB offered enough room to mount the electronic components and routing. However, with the new specifications, it was not possible to use such a simple design to integrate the new components while decreasing the size of the lure. The solution was the use of a Rigid-Flex PCB.

Rigid-Flex PCB is the name given to a PCB that is a combination of both flexible and rigid circuits. Some parts of the PCB can be composed of only flexible materials that can be bent and act as interconnections between the rigid sections, some parts can be flexible but with a solder mask in order to sold some components more easily, and some parts can be rigid to carry all the bulky components and provide a rigid mechanical reference. The number of copper layers can also be varied. Rigid-Flex PCBs give thus the ability to design the circuitry to fit a three-dimensional device in a more optimal way. Indeed, the bulky connectors that are usually used to transfer the information between two PCBs are replaced by flexible surfaces that are parts of the whole PCB and that can bend.

Figure 2 shows the design of the Rigid-Flex PCB made for this study. It is composed of 4 parts that are separated by flexible joints that only contains circuit tracks:

- The head that is rigid and composed of four copper layers. This part contains the eyes that are used to recharge the device (Fig. 2c), the RGB LEDs and their drivers (Fig. 2d), the IR receiver (Fig. 2a) and the battery protection circuits.

- The left fillet that is flexible, composed of two copper layers and a solder mask layer. This part contains the motor drivers (Fig. 2e) and the battery charge circuits.

- The right fillet that is flexible, composed of two copper layers and a solder mask layer. This part contains the microcontroller (Fig. 2b), a voltage sensor to measure the battery voltage and a voltage regulator.

- The tail that is rigid and composed of four copper layers. This part is used to solder the stepper motor (Fig. 2f), connect the programmer (Fig. 2h) and create a contact between the caudal peduncle and the PCB in order to switch ON the device or detect the end position of the caudal peduncle (Fig. 2g).

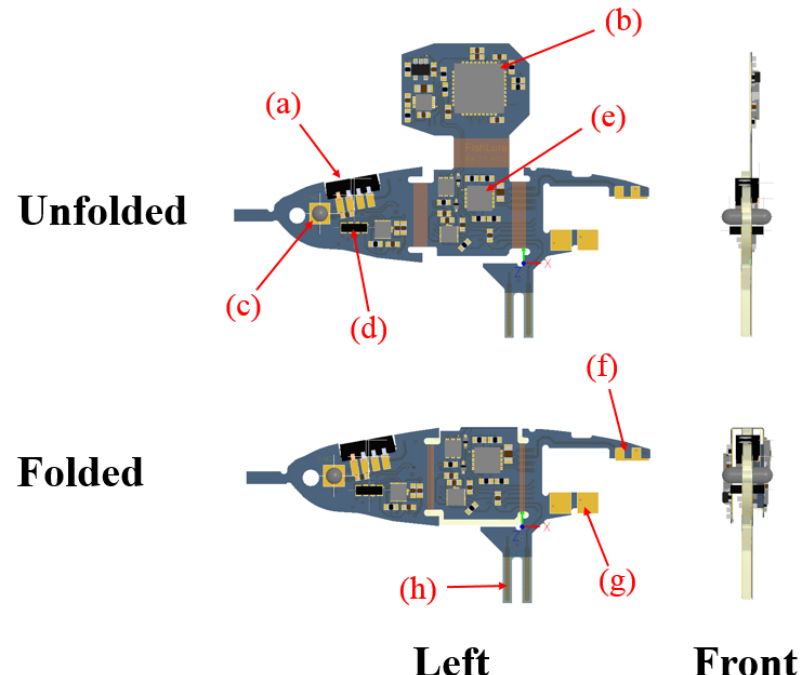

Fig. 2. Top: The PCB design unfolded as it can also be seen in Fig 6 Bottom: The PCB folded. The flexible parts are folded with two 90 degrees angle folds. The blue colored area indicate area with solder mask, while the brown colored indicate the flexible parts without electronic components.

The Rigid parts are $1.2 \mathrm{~mm}$ thick and the flexible parts $0.22 \mathrm{~mm}$. The choice of 4 copper layers for the rigid parts was mandatory for routing purposes. The PCB was manufactured by the company TOM-IC in Switzerland.

\section{B. Power}

We used a miniature Lithium Polymer (LiPo) battery (12x16x5 [mm]) bought from SparkFun (Fig. 3). This battery 
can be recharged through the eyes of the fish that are made of brass and that cross the Polyurethane layer to be accessed from the outside of the device. The battery protection circuits were removed from the original battery and replaced by a home made one on the Rigid-Flex PCB in order to reduce the volume of the battery. A charge circuit was also designed in order to manage the powering of the device and the recharging of the battery.

The caudal peduncle of the fish is made of brass and connected to the ground (GND) (Fig.5g). It can be used for two purposes: It can turn $\mathrm{ON}$ and $\mathrm{OFF}$ the device and also can be used to calibrate the tail position. When the caudal peduncle reaches one of its maximal position, a contact is made between pads on the PCB and the caudal peduncle. When the device is turned OFF and the tail reaches one of the two pads, the microcontroller is switched ON. From this point, the tail is used for the break function implemented in the microcontroller in order to calibrate the tail initial position. Indeed, there are no sensors placed in order to retrieve the position of the stepper motor, and due to the fact that the stepper motor might lose steps, one has to recalibrate sometimes the tail position, and the contact made between pads on the PCB and the tail is used to determine the two maximal position of the tail, and thus the zero position located in the middle of these two extreme positions.

\section{Actuation}

We selected a micro step gear motor MF03G of Seiko Precision Inc. for the actuation of the caudal peduncle. The motor has very small dimensions (Fig. 3) and can thus be easily integrated inside the robotic lure design. The advantage of using a stepper motor is that the motor can be controlled in open-loop and thus no additional sensors to measure the position of the tail is needed.

The original pinion of the stepper motors was removed and replaced by a 3D printed caudal peduncle made of brass for current conductivity as explained in the previous section.

\section{Communication}

IR communication is implemented to remotely control the lure. An IR sensor TSOP75436WTT is located on the head of the lure and IR signal can be sent from any direction to control the device underwater. The protocol RC5 was chosen to transfer information to control the actuator with different frequencies and amplitudes, and the colors and intensities of the LEDs. Thus, a universal television remote control with RC5 protocol could be used to control the robot.

\section{E. LED}

We selected the full-color RGB OVSRRGB LED. This LED is very compact $(3.2 \times 1.5 \times 1[\mathrm{~mm}])$ and has a maximum luminosity intensity of $450 \mathrm{mcd}$. The LED is driven by the triple output I2C chip NCP5623C. The I2C allow us to drive the two LEDs separately using only two circuit tracks (SCL and SDA) from the microcontrollers to the chips. Some studies showed that the presence of realistic eyes on the lure design could increase the acceptance of the fish towards the

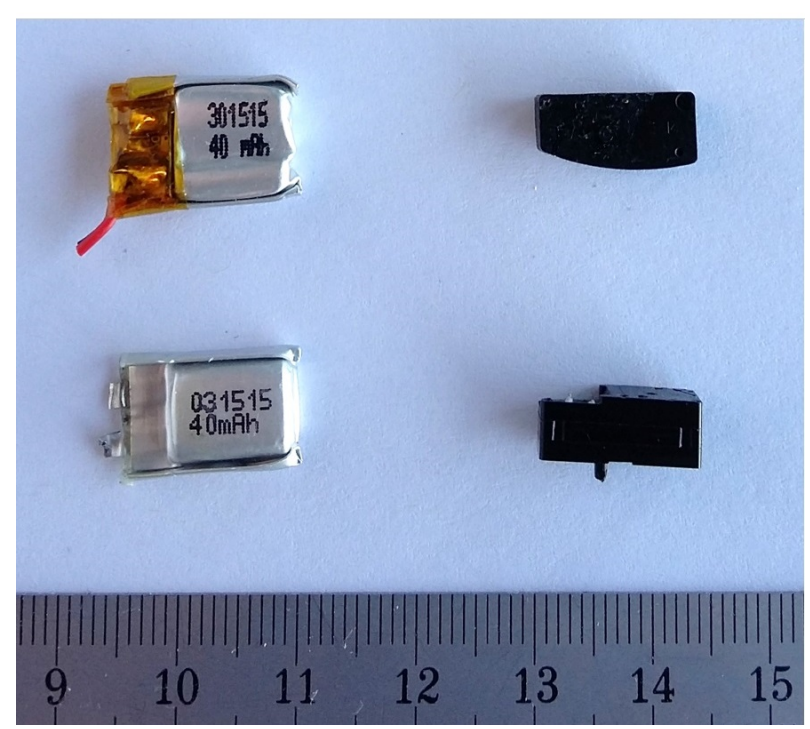

Fig. 3. Left: LiPo battery selected for the design, with the protection circuit (Top) and without the protection circuit (Bottom). In order to reduce the size of the battery, the protection circuit was removed and replaced by one implemented on the Rigid-Flex PCB. Right: top and side views of the Seiko Precision stepper motor used to actuate the tail of the robotic fish (Units in $[\mathrm{mm}])$.

device [3], thus we decided to place one LED near each eye in order to increase the visibility of this part of the body towards the fish.

\section{F. Mechanical design}

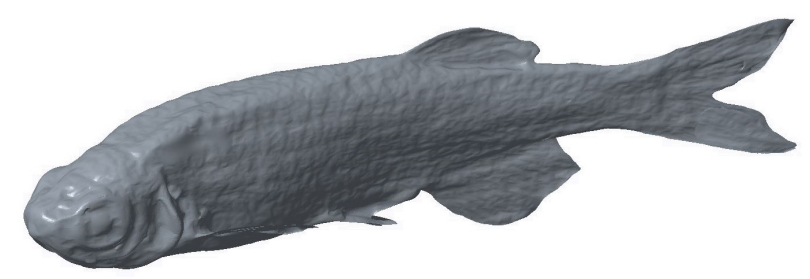

Fig. 4. 3D scan of a male zebrafish Danio Rerio.

The external shape of the lure was defined using the 3D scan of a dead zebrafish Danio Rerio (Fig. 4). The zebrafish was scanned using a Stereoscan 3D Breuckmann StereoSCAN3D with two cameras of 1.4 megapixels. The surfaces retrieved by the scanner were processed and scaled in order to design a mold with the desired undercut of the zebrafish in which the Rigid-Flex PCB could fit. The mold was made of ABS using 3D printing.

The fins of the fish were made using Mylar ${ }^{\circledR}$ as it offers good softness and is available in very thin films. The fins have a thickness of $50 \mu \mathrm{m}$ and were also designed using the 3D scan of the fish.

\section{ASSEMBLY}

\section{A. Component Soldering}

The electronic components were soldered prior to the folding of the PCBs in order to facilitate their positioning. 


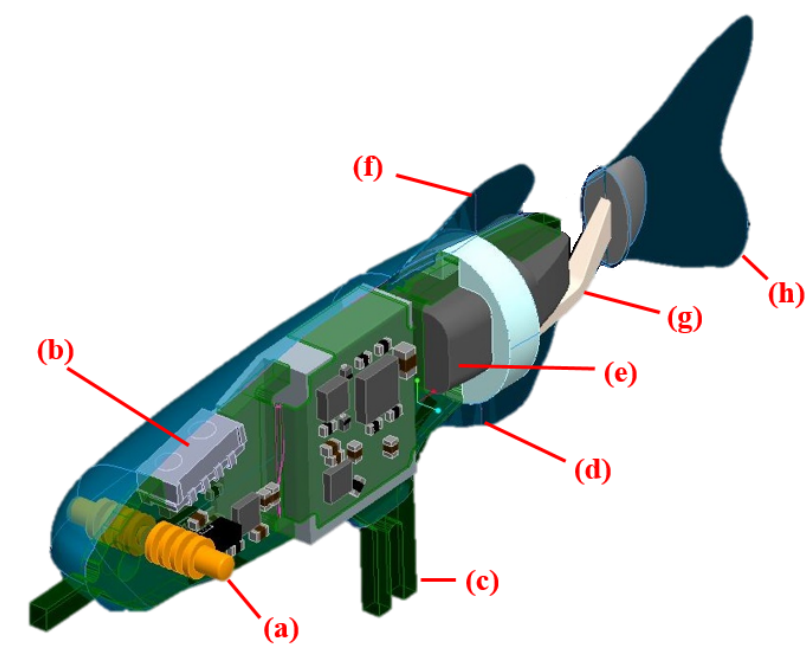

Fig. 5. The mechanical design of the lure was made using PTC CREO Parametric, educational edition. This software allows surface design that could match the 3D scan of the zebrafish. It was used to design the RigidFlex PCB shape, the caudal peduncle, the fins and the molds. a) Eyes. b) IR sensor. c) Contacts to reprogram the device and attach the lure to a module equipped with magnets to be coupled with an external mobile robot. d) Anal fin. e) Stepper motor. f) Dorsal fin. g) Caudal peduncle. h) Caudal fin.

A solder stencil was designed to deposit the solder material, then the PCB was put into a vapor phase oven to solder the electronic components. The eyes, the stepper motors and the battery were soldered as a second phase using a soldering iron.

\section{B. PCB folding}

Figure 7 shows the procedure to fold the PCB. First the PCB is mounted on a support and two metallic wedges are screwed to constrain the PCB and mark the fold (Fig. 7a). The two flexible parts between the left fillet, the head and the tail are folded with two 90 degrees angles (Fig. 7b). This process is very delicate as only one fold can be done, otherwise the flexible part would break, therefore it is irreversible. The battery is then inserted and glued on the left fillet (Fig. 7c). Finally, the right fillet is folded around the battery and glued on it (Fig. 7d).

\section{Tail Molding}

In order to isolate the actuator and the caudal peduncle from water and to create a soft skin that can mimic the tail of the zebrafish, an undercut of the tail was 3D printed and was dipped into liquid latex. The skin created was unmolded and attached on the ring using silicone. The caudal fin, the anal fin and the dorsal fin are made using thin films of Mylar ${ }^{\circledR}$. The Mylar ${ }^{\mathrm{R}}$ is laser cut and then glued on the tail using latex.

\section{Final Molding}

Polyurethane is used to isolate the electronics from water. The mold is composed of two parts that are joined using pins and screws during the molding process to press the two parts against each other. The PCB is placed inside the

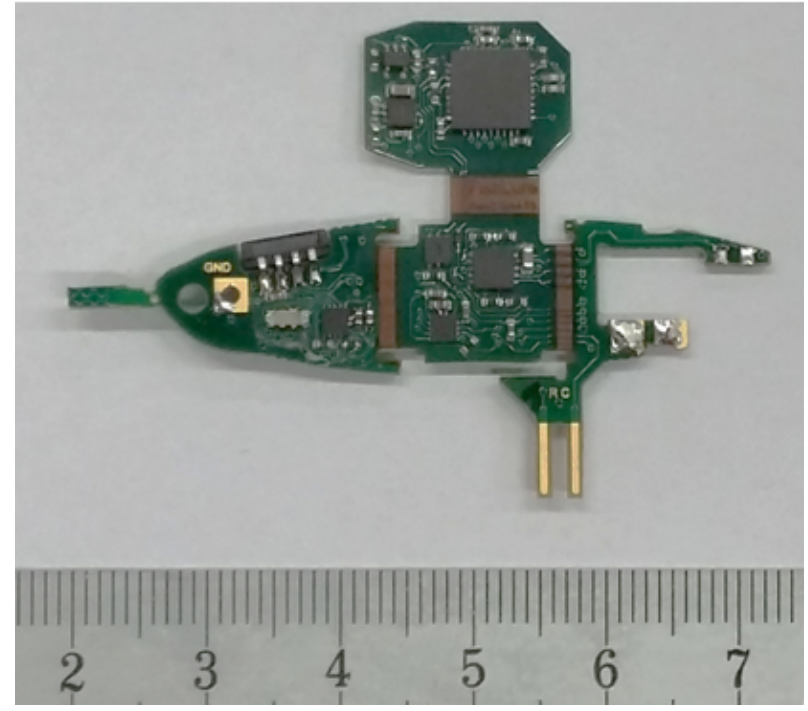

Fig. 6. Prototype of the Rigid-Flex PCB with the electronic components soldered. The head and the tail parts are rigid with two layers of solder mask (green). The left and right fillets are flexible with one layer of solder mask on which the components are soldered. The flexible joints between the parts that contain electronic circuits are colored in brown due to the color of the cover layer as they are not composed of a solder mask (Units in $[\mathrm{mm}])$.

mold and the eyes are used as a reference inside the mold. Finally, liquid polyurethane is injected inside the mold from the tail. The Polyurethane coats the entire PCB up to the ring and hardens inside the mold. After this process, the robotic lure is totally isolated from water and can start to be used underwater (Fig. 8).

\section{VALIDATION}

The first prototype of the robotic fish is presented in Fig. 8. We are really satisfied about the result in term of biomimetics and size. The last aspect that still need to be considered is the color and pattern of the lure. Some lures will be painted in order to mimick the visual aspect of the zebrafish.

We performed a successful remote controlled experiment with the lure below $30 \mathrm{~cm}$ water layer. The waterproofness of the lure was validated. The actuated tail was found to run well, with amplitude measured between 0 and 23 degrees and frequencies between 0 and $20 \mathrm{~Hz}$. We could test the device for up to 23 minutes with the tail of the lure moving continuously using the embedded LiPo battery. The LED could also be well seen through the polyurethane layer.

During the folding process, we encountered an issue on the components that were soldered near the flexible part (Fig. 10). On the design, some electronic components were located very close to the flexible part in order to use all the available surface, thus the distance between the contact pad to solder the components and the flexible part were less than $1 \mathrm{~mm}$. Once folded, the constraint on the electronic circuit in the rigid part were thus too big for the components located very close and thus we encounter breaks either between the pad and the copper layer either between the pin and the pad. 
a)

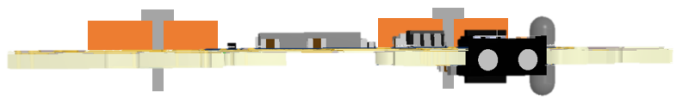

b)

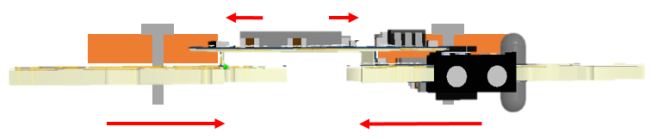

c)

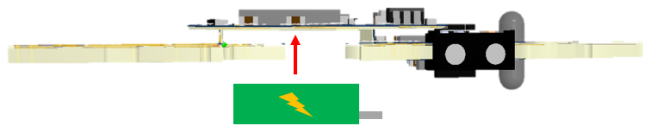

d)

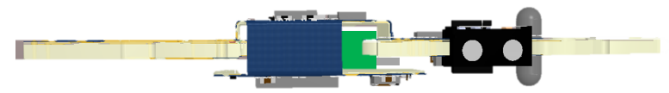

Fig. 7. Folding process of the PCB (Top view). a) Some metallic wedges (in orange) are fixed on the PCB. b) The PCB is folded against the wedges with two 90 degrees angles. c) The battery is placed and soldered onto the PCB. d) The right fillet is folded around the battery and glued on the battery.

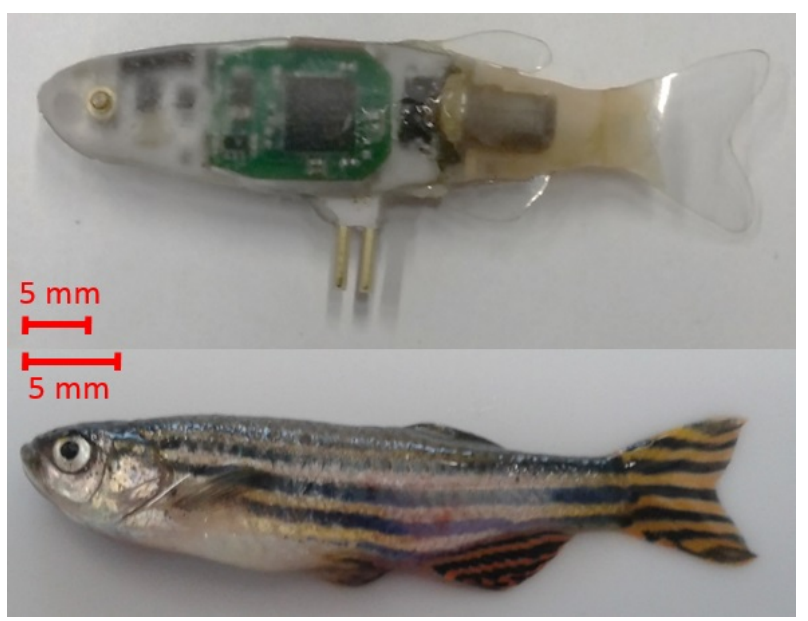

Fig. 8. Top: Final prototype of the robotic fish lure compared with one of our zebrafish Danio Rerio. The latex tail is mounted with the Mylar $\mathbb{R}$ fins. The front part of the lure is molded into polyurethane and only the eyes made of brass are crossing the polyurethane layer. The eyes are used to recharge the device and the four contacts on the pelvic fin are used to reprogram and debug the device.

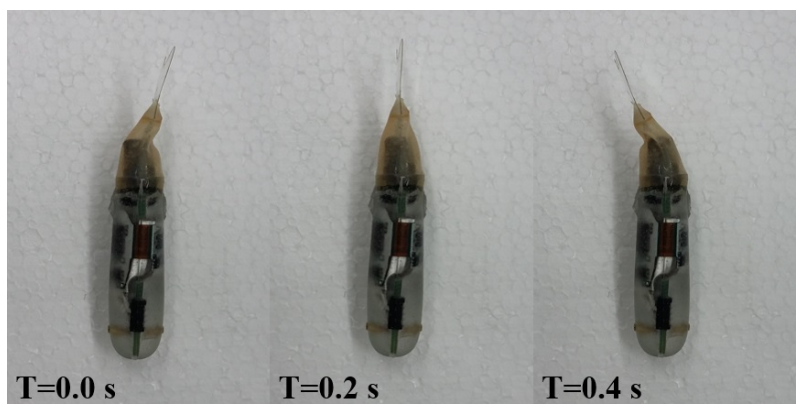

Fig. 9. Top view of the lure with its tail beating with an amplitude of 23 degrees and a frequency of $1.25 \mathrm{~Hz}$.
The solution for this design was to glue the pads in order to rigidify the mechanical connection. We will improve that on a further design, with components located slightly further from the flexible part.

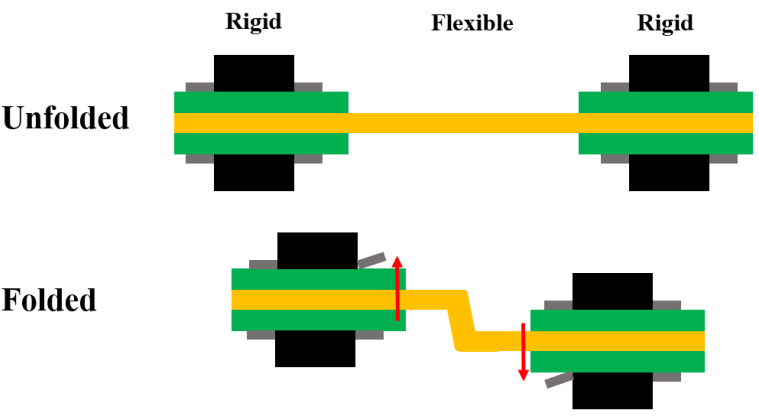

Fig. 10. Top: Rigid-Flex PCB unfolded. The components are soldered on both side of the rigid parts. Bottom: Rigid-Flex PCB folded with the flexible parts bent two times with 90 degrees angles. The side of the components very close to the flexible parts may break during the folding process.

Regarding the improvements on the new design of the robotic fish lure compared with the old one, we describe in Tab. I some of the characteristics to show the differences between the two versions. The maximal length and height were reduced by a factor of 1.26 and 1.42 respectively. The maximal width which is located on the head of the lure was slightly increased as we respected the size ratio. Regarding the PCB surface available for soldering the electronic components and the estimated volume, we see that thanks to the Rigid-Flex PCB technique, we could increase the available surface to mount electronic components by a factor of 1.16 while decreasing the volume of the system with a factor of 1.56. The mass of the new design is also reduce with a factor of 1.77 .

Fig. 11 shows the comparison between the external shape of different designs used in similar research of interaction between zebrafish Danio Rerio and robotic lures [8], and the device designed in this study. We can see that the lure designed in this study is almost half the length of the lure presented in [8]. Moreover, in terms of aspect ratio, Fig. 8 demonstrated that the lure in this study was designed respecting the aspect ratio of a zebrafish as it was based on a 3D scan of a zebrafish that was scaled so that the electronic and mechanical components could fit into, which was not the case in our opinion with the lures used in other similar studies.

TABLE I

COMPARAISON OF SOME CHARACTERISTICS BETWEEN THE OLD AND NEW DESIGN OF THE ROBOTIC FISH LURE.

\begin{tabular}{|l|c|c|}
\hline Version & Old & New \\
\hline Max. length [mm] & 80 & 63 \\
Max. width [mm] & 10 & 11 \\
Max. height [mm] & 20 & 14 \\
PCB surface [mm $\left.{ }^{2}\right]$ & 858 & 1002 \\
Estimated volume $\left[\mathrm{mm}^{3}\right]$ & 8320 & 5390 \\
Mass [g] & 10.48 & 5.9 \\
\hline
\end{tabular}


(a)

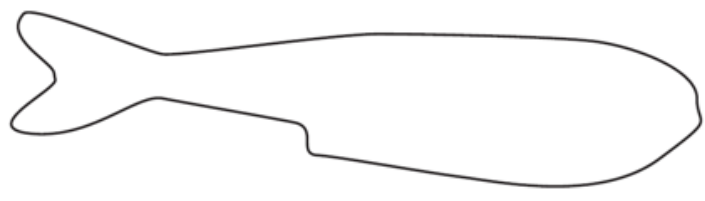

(b)

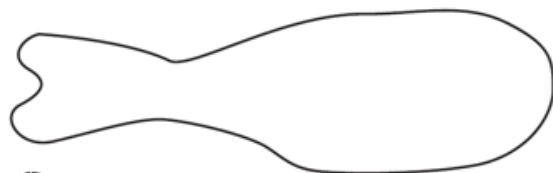

(c)

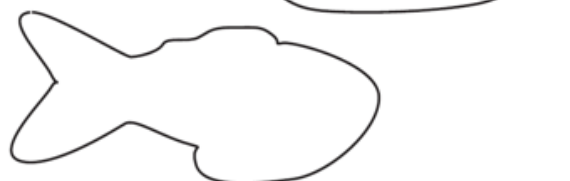

(d)

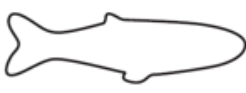

$10 \mathrm{~cm}$

Fig. 11. Comparison between the external shape of lures used in other studies of zebrafish-robot interactions [8] (a)-(c) and the robotic lure designed in this study (d).

In general, the use of Rigid-Flex PCB was really beneficial for the miniaturization of the lure. It allowed us to place more electronic components, reduce the size of the device and mimick most of the body features of a zebrafish. We think that this methodology of using Rigid-Flex PCB methods to fit electronics components into a biomimetics design will help scientists during the miniaturization step of their devices for biomedical robotics and biomechatronics applications.

\section{CONCLUSIONS AND FUTURE WORKS}

We designed, tested and validated an improved version of a robotic fish lure that will be used in the context of social behavior studies with zebrafish Danio Rerio. The robot was found to run well and the reduce mass offer more dynamic movements underwater with the lure coupled with an external mobile robot.

We showed that when using Rigid-Flex PCB techniques, we could overcome the miniaturization of a miniature robotic devices while adding new electronic components. The available surface to mount electronic components could be increased on the new design compared to the previous one that used a rigid PCB and the volume of the system could be decreased. The example was in the context of animalrobot interaction studies, but this can be extended to other domains.

We demonstrated that it is possible to fold flexible PCB with two angles of 90 degrees. However, we have shown the limitation of this system, with electronic components that should be at a certain distance from the folding in order to avoid the rupture of some mechanical contacts.

In further work, we will use this design coupled with an external wheeled mobile robot to allow steering from below the tank in order to perform interaction experiments with living zebrafish Danio Rerio. We will measure the effect of the beating tail coupled with the RGB LEDs on the lure acceptance toward a shoal of fish.

\section{ACKNOWLEDGMENT}

This work was supported by the EU-ICT project ASSISIbf, No. 601074. The information provided is the sole responsibility of the authors and does not reflect the European Commissions opinion. The European Commission is not responsible for any use that might be made of data appearing in this publication. We also thank Marion Segall, Dr. Anthony Herrel and Dr. Ramiro Godoy-Diana for the 3D scan of the zebrafish. Finally, we would like to thank TOM-IC company for producing the Rigid-Flex PCBs.

\section{REFERENCES}

[1] F. Bonnet, S. Binder, M. Elias de Oliveira, J. Halloy and F. Mondada. A Miniature Mobile Robot Developed to be Socially Integrated with Species of Small Fish. IEEE International Conference on Robotics and Biomimetics, Bali, Indonesia, 2014.

[2] P. Phamduy, G. Polverino, R. C. Fuller and M. Porfiri Fish and robot dancing together: bluefin killfish females respond differently to the courtship of a robot with varying color morphs. Bioinspiration and Biomimetics, no. 9, 2014.

[3] T. Landgraf, D. Bierbach, H. Nguyen, N. Muggelberg, P. Romanczuk and J. Krause, RoboFish: increased acceptance of interactive robotic fish with realistic eyes and natural motion patterns by live Trinidadian guppies Bioinspiration and Biomimetics, no. 1, 2016.

[4] J. Faria, J. Dyer, R. Clément, I. Couzin, N. Holt, A. Ward, D. Waters, and J. Krause, A novel method for investigating the collective behaviour of fish: Introducing Robofish, Behavioral Ecology and Sociobiology, vol. 64, pp. 1211-1218, 2010.

[5] D. T. Swain, I. D. Couzin, and N. E. Leonard, Real-time feedbackcontrolled robotic fish for behavioral experiments with fish schools. Proceedings of the IEEE, vol. 100, no. 1, pp. 150163, 2012.

[6] M. Worm, T. Landgraf, H. Nguyen, G. von der Emde, Electrocommunicating Dummy Fish Initiate Group Behavior in the Weakly Electric Fish Mormyrus rume, Biomimetic and Biohybrid Systems, vol. 8608, pp 446-448, 2014

[7] S. Marras and M. Porfiri, Fish and robots swimming together: attraction towards the robot demands biomimetic locomotion, J. R. Soc. Interface, Aug 7;9(73):1856-68, 2012.

[8] N. Abaid, T. Bartolini, S. Macri, and M. Porfiri, Zebrafish responds differentially to a robotic fish of varying aspect ratio, tail beat frequency, noise and color, Behavioural Brain Research, vol. 233, pp. 545-553, 2012.

[9] F. Mondada, A. Martinoli, N. Correll, A. Gribovskiy, J. I. Halloy, R. Siegwart, and J.-L. Deneubourg. A general methodology for the control of mixed natural-artificial societies. In S. Kernbach, editor, Handbook of Collective Robotics, Chapter 15, pages 547-586. Pan Stanford Publishing, Singapore, 2013.

[10] D. J. Hoare, I. D. Couzin, Godin, and J. Krause, Context-dependent group size choice in fish, Animal Behaviour, vol. 67, no. 1, pp. 155164, Jan. 2004.

[11] G. G. Rosenthal and M. J. Ryan, Assortative preferences for stripes in danios, Animal Behaviour, vol. 70, no. 5, pp. 1063 1066, 2005.

[12] C. Saverino and R. Gerlai, The social zebrafish: Behavioral responses to conspecific, heterospecific, and computer animated fish, Behavioural Brain Research, vol. 191, no. 1, pp. 77 87, 2008.

[13] F. Bonnet, Y. Kato, J. Halloy and F. Mondada. Infiltrating the Zebrafish Swarm: Design, Implementation and Experimental Tests of a Miniature Robotic Fish Lure for Fish-Robot Interaction Studies,. SWARM 2015: The First International Symposium on Swarm Behavior and Bio-Inspired Robotics, Kyoto, Japan, 2015.

[14] R. Spence, G. Gerlach, C. Lawrence, and C. Smith, The behaviour and ecology of the zebrafish, danio rerio, Biological Reviews, vol. 83, no. 1, pp. 1334, 2008. 